\title{
Electronic conduction in multi-walled carbon nanotubes: Role of intershell coupling and incommensurability
}

\author{
Stephan Roche ${ }^{\ddagger}$, François Triozon ${ }^{\dagger}$, Angel Rubio * and Didier Mayou ${ }^{\dagger}$ \\ ${ }_{\ddagger}$ Commissariat à l'Énergie Atomique, DRFMC/SPSMS, Grenoble, France. \\ † LEPES-CNRS, avenue des Martyrs BP166, 38042 Grenoble, France. \\ * Departamento de Física de Materiales, Facultad de Ciencias Químicas, Universidad del Pais \\ Vasco/Euskal Herriko Unibertsitatea, Apdo. 1072, 20018 San Sebastián/Donostia, Basque \\ Country, Spain.
}

\begin{abstract}
Geometry incommensurability between weakly coupled shells in multi-walled carbon nanotubes is shown to be the origin of unconventional electronic conduction mechanism, power-law scaling of the conductance, and remarkable magnetotransport and low temperature dependent conductivity when the dephasing mechanism is dominated by weak electron-electron coupling.
\end{abstract}


Single-walled carbon nanotubes (SWNTs) can be either metallic of semiconducting depending on their helicities, i.e. how the graphene sheet is rolled upla. For weak uniform disorder, the application of the Fermi golden rule for metallic SWNTs have demonstrated $\mu m$ long mean free path\$2 in agreement with experiments, clearly pointing towards ballistic transport是目.

Multi-walled carbon nanotubes (MWNTs) typically consist of several tenths of coaxial shells with intershell coupling between neighboring layers. The understanding of intrinsic properties of MWNTs remain elusive, since different transport scenario have been reported from ballistic, diffusive and low resistive, to insulating behaviors 6 - An underestimated feature of MWNT's geometry is that depending on their helical vector $(n, m)$ (expressed in the basis of the honeycomb lattice), two consecutive shells are commensurate (resp. incommensurate), if the ratio between their respective unit cell lengths $T_{(n, m)}$ along the tube axis is a rational (resp. irrational) numbert. An illustrative case is given on Fig.1 where the two double-walled nanotubes $(9,0) @(18,0)$ and $(9,0) @(10,10)$ are shown. Apparently, these defect-free structures should conduct electrons in a similar fashion since they are constituted of metallic shells with nearly identical diameters. Notwithstanding, by introducing coupling between shells, the $(9,0) @(18,0)$ remains a periodic system, with a unit cell of length $T_{(9,0)}=T_{(18,0)} \sim 2.15 \AA$, whereas the $(9,0) @(10,10)$ can not be described by a single cell, since $T_{(10,10)} / T_{(9,0)}=\sqrt{3}$, so that coupling strengths between nearest-neighbors carbon atoms located at different shells, are not translationally invariant along the tube axis. The system is therefore not-periodic and electronic wave-coherence is expected to be qualitatively different. Incommensurate structures have an increasingly higher probability to be synthetized than fully commensurate structures, when increasing the number of inner shells.

Incommensurability was recently shown to yield anomalous corrugation properties 9 . Non-ballistic conduction was reported and implications on magnetotransport discussed 0 . Hereafter after defining the hamiltonian together with the computational method used, several consequences of the non-ballistic conduction are analyzed for transport coefficients in MWNTs. 
Hamiltonian parameters for the MWNTs.-Our tight-binding model features one $p_{\perp^{-}}$ orbital per carbon atom, zero onsite energies, and constant nearest-neighbor hopping on each layer n (n.n.), and hopping between neighboring layers (n.l.) are defined by 1 :

$$
\mathcal{H}=\gamma_{0}\left[\sum_{i, j n . n .}\left|p_{\perp}^{j}\right\rangle\left\langle p_{\perp}^{i}\right|\right]-\beta\left[\sum_{i, j \in n . l .} \cos \left(\theta_{i j}\right) e^{\frac{d_{i j}-a}{\delta}}\left|p_{\perp}^{j}\right\rangle\left\langle p_{\perp}^{i}\right|\right]
$$

where $\theta_{i j}$ is the angle between the $p_{\perp}^{i}$ and $p_{\perp}^{j}$ orbitals, and $d_{i j}$ denotes their relative distance. The parameters used here are : $\gamma_{0}=2.9 \mathrm{eV}, a=3.34 \AA, \delta=0.45 \AA$. Estimate based on abinitio calculations for $\beta$ gives $\beta \simeq \gamma_{0} / 8$, but in order to get insight in the effect of $\beta$ on transport properties (since small change in the interlayer distance yield large variation of the coupling strength), several values of $\beta\left(0 \leq \beta \leq \gamma_{0}\right)$ have been considered. Synthetized MWNTs contain typically a few tenth of inner layers, but here the study is restricted to 2 and 3-walled nanotubes, taking the intershell distance of $3.4 \AA$ as in graphite.

Quantum diffusion in MWNTs.-We investigate quantum dynamics through the propagation of wavepackets in long MWNTs with about 1000 unit cells $(\sim$ half million of carbon atoms). The time-dependent Schrödinger equation is solved numerically by using a polynomial expansion of the evolution operator. It gives us access to the diffusion coefficient of a wavepacket $|\psi\rangle$. If $|\psi\rangle$ is initially localized at the center $(\mathrm{x}=0)$ of the nanotube, then its diffusivity is simply $D_{\psi}(t)=L_{\psi}(t)^{2} / t$ with $L_{\psi}(t)=\sqrt{\left\langle\psi\left|(\hat{X}(t)-\hat{X}(0))^{2}\right| \psi\right\rangle}$ the spatial spreading of the wavepacket ( $\hat{X}$ is the position operator along the tube axis), and the system size is taken sufficiently large to avoid boundary effects. For random-phase or energyfiltered initial states extented to the whole system 12 , periodic boundary conditions are used for the tube of length $\mathcal{L}$, and the diffusivity is approximated as $D_{\psi}(t) \simeq 4 \pi^{2} / \mathcal{L}^{2} I_{\psi}(t) / t$, where $I_{\psi}(t)=\left\langle\psi\left|\hat{A}^{+}(t) \hat{A}(t)\right| \psi\right\rangle$ and $\hat{A}(t)=\exp (2 i \pi \hat{X}(t) / \mathcal{L}) \exp (-2 i \pi \hat{X}(0) / \mathcal{L})-\mathbb{1}$. This estimation of $D_{\psi}(t)$ is accurate only for diffusion lengthes smaller than $\mathcal{L}$, as checked by finite-size scaling analysis. The average spreading $L(t)$ and the average diffusion coefficient $D(t)$ are defined by $L(t)=\sqrt{\left\langle L_{\psi}^{2}(t)\right\rangle}=\sqrt{t D(t)}$, where \langle\rangle denotes an average over many 
initial wavepackets. $D\left(\tau_{\varphi}\right)$ is the average diffusivity along the nanotube axis, if at $\tau_{\varphi}$ the electronic wavefunction looses its phase memory due to some inelastic scattering. The diffusion coefficient at $\tau_{\varphi}$ are thus connected to the Kubo conductivity $\sigma=e^{2} \rho D\left(\tau_{\varphi}\right)$, where $\rho$ is the density of states. This approach provides a good qualitative picture of wavepacket propagation in MWNTs constituted of conducting shells, with energies away from the charge neutrality point. Experimental studies demonstrate that chemical potential away from the charge neutrality point can be obtained upon doping 13 . Krüger et al. 5 obtained variations of the Fermi energy of the order of $\sim 0.3 \mathrm{eV}-0.5 \mathrm{eV}$, corresponding to $10-15$ conducting channels instead of 2 per metallic layer.

Anomalous conduction in incommensurate MWNTs.-For disorder-free commensurate systems, a wavepacket initially localized in the outermost shell of the MWNTs, quickly transfer its weight to inner shells, due to intershell coupling. By following its spreading properties, we found that the wavepacket propagation is ballistic along the tube-axis over large distances $(\mu \mathrm{m})$, i.e. $L(t) \sim v t$ similarly to the case of perfect metallic single-walled nanotube.

However, for the incommensurate case, the transfer of the electronic wavepacket is followed by a non ballistic propagation given by $L(t) \sim \mathcal{A} t^{\eta}$. The coefficient $\eta$ is found to decrease from $\sim 1$ to $\sim 1 / 2$ by increasing the number of coupled incommensurate shells 1 . On Fig.2, ballistic versus non ballistic conduction mechanisms are illustrated in the defectfree incommensurate double-walled nanotubes $(9,0) @(18,0)$ and $(9,0) @(10,10)$. The case $(6,4) @(10,10) @(17,13)$ is also shown to demonstrate the effect of increasing the "strength" of incommensurability on the wavepacket propagation. The interlayer coupling parameter taken here is given by $\beta=\gamma_{0} / 3$ which is slightly larger than the expected ab-initio value. By increasing $\beta$, a reduction of power-law exponent $\eta$ is obtained, similarly to what is found by increasing the number of coupled incommensurate shells, for a fixed value of $\beta$.

Magnetotransport properties.-The effect of magnetic field and disorder when charges propagate predominantly on the outermost shell has been analyzed in 10 . Here, we focus on the magnetotransport in incommensurate MWNTs if the magnetic field is applied parallel to the tube axis. Magnetic field dependent diffusion coefficients of electronic wavepackets are 
given on Fig.3., for incommensurate 2-wall $(9,0) @(10,10)$ (main frame) in the power-law diffusion regime (with two values of $\beta=\gamma_{0} / 3, \gamma_{0}$ ). $\Phi_{0}$-periodic oscillations and positive magnetoresistance at low field are observed, similar to what is predicted for the ballistic regime in the SWNT case 1 . Note that the Aharonov-Bohm oscillations for the commensurate $(9,0) @(18,0)$ are still $\Phi_{0}$-periodic, but with much smaller amplitude (not shown here). By increasing $\beta$, the coupling strength between walls, the magnetoconductance is reduced without modification of the oscillatory behavior.

Differently, for the incommensurate nanotube $(6,4) @(10,10) @(17,13)$ (inset of Fig.3), in which diffusive-like propagation takes place, there is evidence for negative magnetoresistance at low field and $\Phi_{0} / 2$-periodicity of $D\left(\tau_{\phi}, \Phi\right)$. From the saturation of diffusivity, we deduce some effective elastic mean free path $\tilde{l}_{e}$, that turns out to be slightly smaller that the outer shell circumference (for $\beta=\gamma_{0}$ ). By considering a reduced value of the coupling parameter $\left(\beta=\gamma_{0} / 3\right.$-not shown here), despite the fact that the effective mean free path becomes larger than the outershell circumference, still negative magnetoresistance at low field and $\Phi_{0} / 2$-periodic oscillation persist. These results confirm that magnetotransport properties of MWNTs are very sensitive to the geometry, the number of shells carrying current, and the hamiltonian parameters. In disordered systems, the basic scheme is that of ballistic electrons scattering on random impurities. The $\Phi_{0} / 2$ periodicity showed here, also results from quantum interferences of the electronic pathways around the cylinder wrist. However the usual scheme of interfering backscattered electronic trajectories is not strictly applicable for incommensurate disorder-free MWNTs, as confirmed by a study of the probability of return to the origin of wavepackets of magnetoconductance and gave an interpretation in terms of superposition of phase shift due to spectral effects, assuming three internal tubes with different helicity (same situation as in $(6,4) @(10,10) @(17,13))$. The effect of magnetic field on density of states (DoS) has been discussed theoretically 19 and should also be considered to fully understand the magnetotransport in SWNTs and moderately large diameter MWNTs.

Electron-electron interactions and temperature dependence of inelastic scattering times 
and conductivity-.We focus now on the combined effect of anomalous propagation of wavepackets (ASWP) together with a dephasing mechanism dominated by electron-electron interactions. Recently, the influence of ASWP on frequency and temperature dependences of inelastic scattering times and conductivities, in systems close to a metal-insulator transition 20, in aperiodic incommensurate and quasiperiodic structures 2123 has been demonstrated.

The effect of electron-electron interaction in transport properties of MWNTs can be addressed within two limits. The strong coupling case is related to the existence of a Luttinger liquid phase, which has been discussed theoretically recently for metallic commensurate MWNTs4 The effect of incommensurability on Luttinger liquid was shown in other context to develop new phase diagram 25, result that could be valuable in the context of incommensurate carbon nanotubes. In the weak coupling limit, the electron-electron interaction acts as the main dephasing mechanism and monitor the temperature dependences of inelastic scattering times and conductivity.

The interference pattern between two waves $\Psi_{1}(t)$ and $\Psi_{2}(t)$ which propagate coherently within the system is obtained from the evaluation of the relative phase difference accumulated in time. In the limit of low temperature, the inelastic coupling with other electrons, which produces a loss of phase coherence, can be expressed by an external time-dependent potential $V\left(L_{\Psi}(t), t\right)$, whose effect is to superimpose new phase factors

$$
\left\langle e^{i \phi}\right\rangle=\left\langle e^{i\left(\int V\left(L_{\Psi}(t), t\right) d t / \hbar\right)}\right\rangle=\int \mathcal{P}(\phi) e^{i \phi} d \phi
$$

with $\phi$ defined by a probability distribution $\mathcal{P}(\phi)$. As the potential is time-dependent, the initial phase interference pattern is reduced in amplitude since the phase factors are modified by uncorrelated phase shifts. The loss of phase coherence has been shown to be driven by the increase of phase uncertainty between the superimposed phases accumulated by the two non-interacting waves $\Psi_{1}(t)$ and $\Psi_{2}(t)$ and related (under certain approximation, see 26 ) to $\left\langle\delta \phi_{1-2}^{2}\right\rangle=\left\langle\phi_{1-2}^{2}\right\rangle-\left\langle\phi_{1-2}\right\rangle^{2}\left(\phi_{1-2}=\int d t V\left(L_{\Psi_{1}}(t), t\right)-V\left(L_{\Psi_{2}}(t), t\right)\right)$ with $V\left(L_{\Psi}(t), t\right)=$ 
$-\frac{e}{c} \frac{d L_{\Psi}(t)}{d t} \cdot \mathcal{A}\left(L_{\Psi}(t), t\right)$, where $\mathcal{A}\left(L_{\Psi}(t), t\right)$ is the potential vector. The inelastic phase coherence time $\tau_{\varphi}$ defines the limit of coherent phase interference and thus comes out from 26

$$
\left\langle\delta \phi_{1-2}^{2}\right\rangle \simeq e^{2} k_{B} T \int_{0}^{\tau_{\varphi}} d t\left|L_{\Psi_{1}}(t)-L_{\Psi_{2}}(t)\right|^{2-d} \simeq 1
$$

In a diffusive medium, $\left|L_{\Psi_{1}}(t)-L_{\Psi_{2}}(t)\right| \sim t^{1 / 2}$, which triggers $\tau_{\varphi}^{-1} \sim T^{2 / 3}$, behavior clearly identified experimentally at low temperature $(T<4 K)$ in metallic wires27.

In defect-free incommensurate MWNTs, ASWP implies a power-law dependent diffusivity $D\left(\tau_{\varphi}\right) \sim \tau_{\varphi}^{2 \eta-1}$, which thus results in a $\tau_{\varphi}^{-1} \sim T^{1 /(1+\eta)}$ power-law for the inelastic scattering times. Subsequently, an anomalous temperature dependence of the conductivity follows

$$
\sigma(T) \sim T^{\frac{(1-2 \eta)}{(1+\eta)}}
$$

In the main frame of Fig.4 such behavior is exemplified for different exponents $\eta$. One notes that a temperature independent conductivity is found for the limit $\eta \rightarrow 1 / 2$ (diffusivelike motion), and the decrease of conductivity with temperature may be completely opposite for subdiffusive regime $(\eta<1 / 2)$. Although in our calculations $\eta$ remains above $1 / 2$, such possibility, often found in quasiperiodic systems, can not be fully discarded for more complex situations. Recently Natelson et al.28 have reported a continuous change of the power law exponent of the inelastic scattering times $\tau_{\varphi}$ by varying the geometry of a quantum wire, i.e. by tuning quantum interferences. Here, we have ascribed similar behavior to the anomalous propagation steming from incommensurability in MWNTs. Along the same lines, one also notes that a study of the frequency dependent conductivity could be an interesting way to unveil anomalous quantum diffusion 23. Frequency dependent study of electronic impedance has been recently demonstrated to be relevant for carbon nanotubes 29 .

Anomalous scaling of the conductance-.The relation between the electronic conductivity and the conductance allows to anticipate an anomalous length dependence of the Landauer conductance. Indeed, in a non-ballistic conduction, electrons propagate according 
$L(t) \sim \mathcal{A} t^{\eta}$, with $\mathcal{A}$ and $\eta$ depending on the MWNT geometry but also the number of shells participating in transport $\left(N_{c}\right)$. The conductance is related to the conductivity through $G(L) \sim \sigma(L) / L$. By defining $D_{n b}(L)$ (resp. $\left.D_{b}(L)\right)$ the value of the non-ballistic diffusion coefficient (resp. ballistic diffusion coefficient for a fully commensurate metallic MWNT with $N_{c}$ shells), then the conductance at scale $L$ writes

$$
\begin{aligned}
\frac{G_{n b}(L)}{2 N_{c} e^{2} / h} & \sim \frac{D_{n b}(L)}{D_{b}(L)} \\
G_{n b}(L) & \sim \frac{2 N_{c} e^{2}}{h} \times \alpha \times L^{(\eta-1) / \eta}
\end{aligned}
$$

with a factor $\alpha \sim\left(\mathcal{A} / v_{F}\right)^{1 / \eta}$ that will depend on the particular system and chemical potential considered. Indeed, if the chemical potential is close to the charge neutrality point, fewer conducting channels will be available because of the presence of semiconducting shells weakly conducting at such energy. The ballistic case $\eta=1$ corresponds to $G \sim \frac{N_{c} e^{2}}{h}$, that is a length-independent conductance, whereas in the diffusive limit, the conductance scales as $\sim 1 / L$ (physically related with the distance between electrodes). To test such physical effect, experiments should be carried out on highly purified MWNTs with typical diameter of $\sim 10-20 \mathrm{~nm}$, since it will allow to tune the participation in transport of more than 10 shells, under reasonably weak chemical doping $(\leq 1 \%)$, or directly by varying the applied voltage $V_{\text {bias }}$ (the number of shells $N_{C}$, participating in conduction being a function of $\left.V_{\text {bias }}\right)$. Furthermore, the study of the conductance with the distance between leads probing the sample requires perfect ohmic contacts between electrodes and the nanotube.

Conclusions-Incommensurability in MWNTs is a geometrical degree of freedom at the origin of non ballistic conduction. The redistribution of the wavepacket in the whole object is followed by a specific multiple wave scattering that jeopardize ballistic conduction. All transport coefficients should be sensitive to such effects, especially the temperature dependence of inelastic scattering times and conductivity driven by electron-electron interactions at low temperature, as well as the length-scaling of the Landauer conductance. Experiments should be performed in conjunction with theoretical calculations of the relevant microscopic parameters $(\mathcal{A}, \eta)$ that depend on the geometry of the MWNT as well as the number of 
shells participating in conduction.

Acknowledgments: Financial support from NAMITECH [ERBFMRX-CT96-0067(DG12MITH)], DGES (PB98-0345), COMELCAN( HPRN-CT-2000-00128), JCyL (VA28/99) and C are acknowledged. S.R. is indebted to Riichiro Saito and Hélène Bouchiat for stimulating discussions. 


\section{REFERENCES}

‡ To whom correspondence should be addressed. E-mail: sroche@cea.fr

${ }^{1}$ R. Saito, G. Dresselhaus, and M. S. Dresselhaus, Physical Properties of Carbon Nanotubes (Imperial College Press, London, 1998).

${ }^{2}$ C.T. White and T. N. Todorov, Nature 393, 240 (1998).

${ }^{3}$ M. Bockrath et al., Science 275, 1922 (1997).

${ }^{4}$ A. Bachtold et al., Phys. Rev. Lett. 84, 6082 (2000).

${ }^{5}$ R. Martel, T. Schmidt, H. Shea, T. Hertel and Ph. Avouris, Appl. Phys. Lett. 73, 2447 (1998). S. J. Tans, R. M. Verschueren, and C. Dekker, Nature 393, 49 (1998).

${ }^{6}$ T.W. Ebbessen et al., Nature 382, 54 (1996). A. Yu Kasumov et al., Europhysics Letters 43, 89 (1998).

${ }^{7}$ S. Frank, P. Poncharal, Z.L. Wang, W. A. de Heer, Science 280, 1744 (1998).

${ }^{8}$ C. Schönenberger, A. Bachtold, C. Strunk and J.P. Salvetat, Appl. Phys. A 69, 283 (1999).

${ }^{9}$ A.N. Kolmogorov and V.H. Crespi, Phys. Rev. Lett. 854727 (2000).

${ }^{10}$ S. Roche, F. Triozon, A. Rubio and D. Mayou, submitted for publication.

${ }^{11}$ R. Saito, G. Dresselhaus, and M. S. Dresselhaus, J. Appl. Phys. 73, 494 (1993). J.C. Charlier and J.P. Michenaud, Phys. Rev. Lett. 70, 1858 (1993). Ph. Lambin, V. Meunier and A. Rubio, Phys. Rev. B 62, 5129 (2000).

${ }^{12}$ F. Triozon, S. Roche and D. Mayou, RIKEN Review 29, 73 (2000).

${ }^{13}$ P.G. Collins, K. Bradley, M. Ishigami, and A. Zettl, Science 287, 1801 (2000).

${ }^{14}$ E. Jouguelet, C. Mathis, P. Petit, Chemical Physics Letters 318, 561 (2000).

${ }^{15}$ M. Krüger, M.R. Buitelaar, T. Nussbaumer, C. Schönenberger, and L. Forró, Appl. Phys. 
Lett. 78, 1291 (2001).

16 T. Ando, Semicond. Sci. Technol. 15, R13 (2000).

${ }^{17}$ F. Triozon and S. Roche, unpublished.

${ }^{18}$ A. Fujiwara, K. Tomiyama, H. Suematsu, M. Yumura and K. Uchida, Phys. Rev. B. 60, 13492 (1999).

${ }^{19}$ H. Ajiki and T. Ando, J. Phys. Soc. Japan 62, 1255 (1993). S. Roche, G. Dresselhaus, M. Dresselhaus and R. Saito, Phys. Rev. B 62, 16092 (2000).

${ }^{20}$ T. Brandes, L. Schweitzer and B. Kramer, Phys. Rev. Lett. 72, 3582 (1994).

${ }^{21}$ M. Takahashi, Y. Hatsugai and M. Kohmoto, J. Phys. Jap. 65, 529 (1996).

${ }^{22}$ S. Roche and T. Fujiwara, Phys. Rev. B 58, 11338 (1998).

${ }^{23}$ D. Mayou, Phys. Rev. Lett. 851290 (2000).

${ }^{24}$ R. Egger, Phys. Rev. Lett. 835547 (1999).

25 J. Vidal, D. Mouhanna and T. Giamarchi, Phys. Rev. Lett. 833908 (1999).

${ }^{26}$ A. Stern, Y. Aharonov and Y. Imry, Phys. Rev. A 41, 3436 (1990).

${ }^{27}$ S. Wind et al., Phys. Rev. Lett. 57, 633 (1986).

${ }^{28}$ D. Natelson, R.L. Willett, K.W. West and L.N. Pfeiffer, Phys. Rev. Lett. 861821 (2001).

${ }^{29}$ Y.-P. Zhao, B. Q. Wei, P. M. Ajayan, G. Ramanath, T.-M. Lu, G.-C. Wang, A. Rubio and S. Roche, submitted for publication. 


\section{Figure captions:}

Figure 1: Geometrical representation of $(9,0) @(18,0)$ (right) and $(9,0) @(10,10)($ left $)$ double-walled carbon nanotubes ASK me by e-mail ! at sroche@cea.fr.

Figure 2: Main Frame: Averaged diffusion coefficient (arb. unit) for $(9,0) @(18,0)$ and $(9,0) @(10,10)$ with $\beta=\gamma_{0} / 3$. Inset: Avergared diffusion coefficient for the incommensurate $\operatorname{MWNT}(6,4) @(10,10) @(17,13)$. One notes that $T_{(6,4)}=3 \sqrt{19} a_{c c}, T_{(10,10)}=\sqrt{3} a_{c c}$, and $T_{(17,13)}=3 \sqrt{679} a_{c c}$ where $a_{c c}=1.42 \AA$ is the interatomic distance between carbon atoms.

Figure 3: Main frame : Magnetic field dependence of the diffusion coefficient for the incommensurate double-walled $(9,0) @(10,10)$ for two values of $\beta=\gamma_{0} / 3$ (solid line, right $\mathrm{y}$-axis) and $\beta=\gamma_{0}$ (dashed line, left $\mathrm{y}$-axis) evaluated at the same time $\tau_{\phi}$. Inset : Case for $(6,4) @(10,10) @(17,13)$ at two different times $\tau_{\phi}\left(\hbar / \gamma_{0}\right.$ unit $)$ for $\beta=\gamma_{0}$.

Figure 4:Main Frame: Scaling of the temperature dependence of the conductivity with decreasing exponent $\eta$. Inset: Length dependence of the conductance for the same parameters. Bold solid lines are for $\eta \sim 1$, and bold dashed lines for $\eta \sim 1 / 2$, whereas other solid lines interpolate between 1 and $1 / 2$. 


\section{FIGURES}

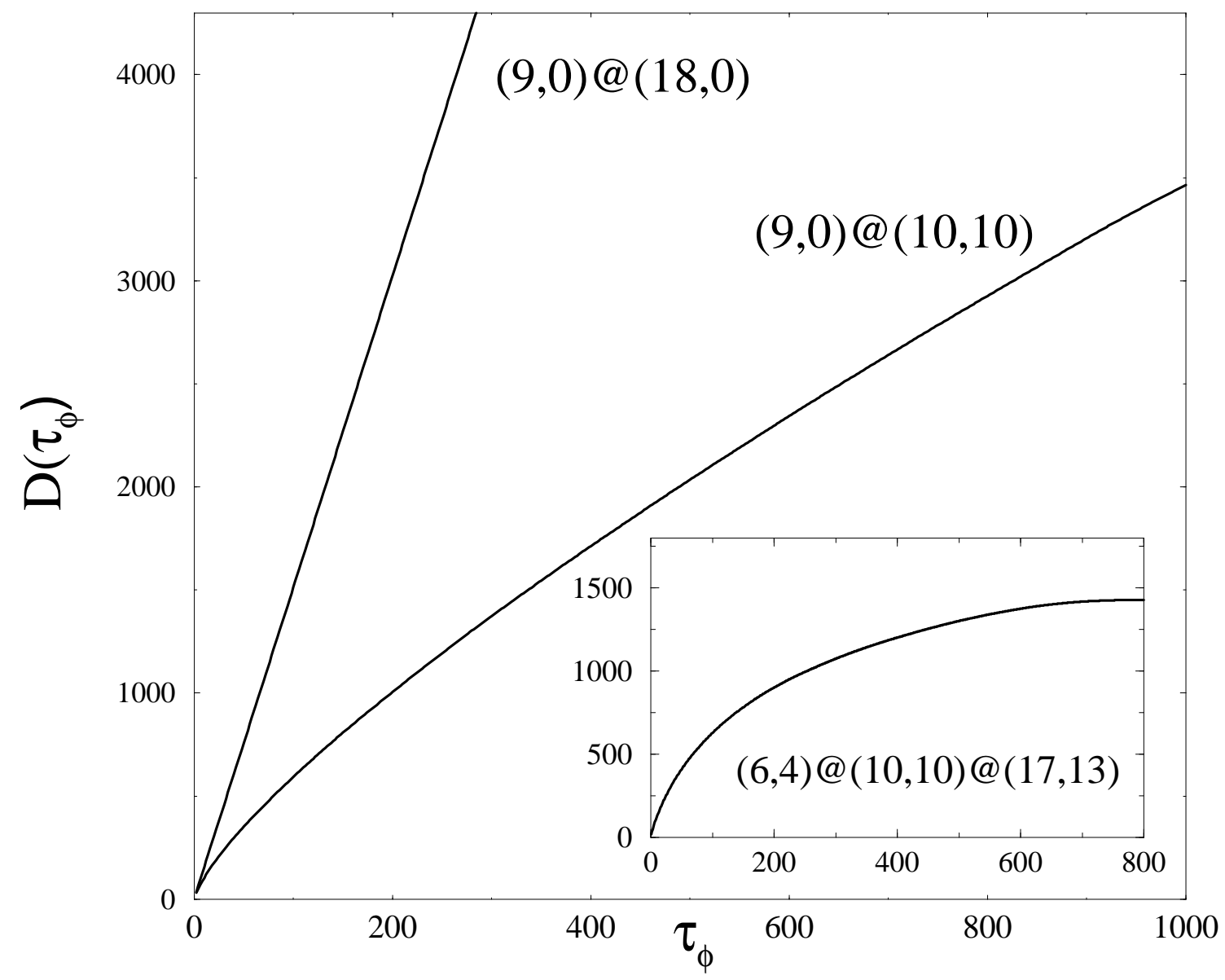




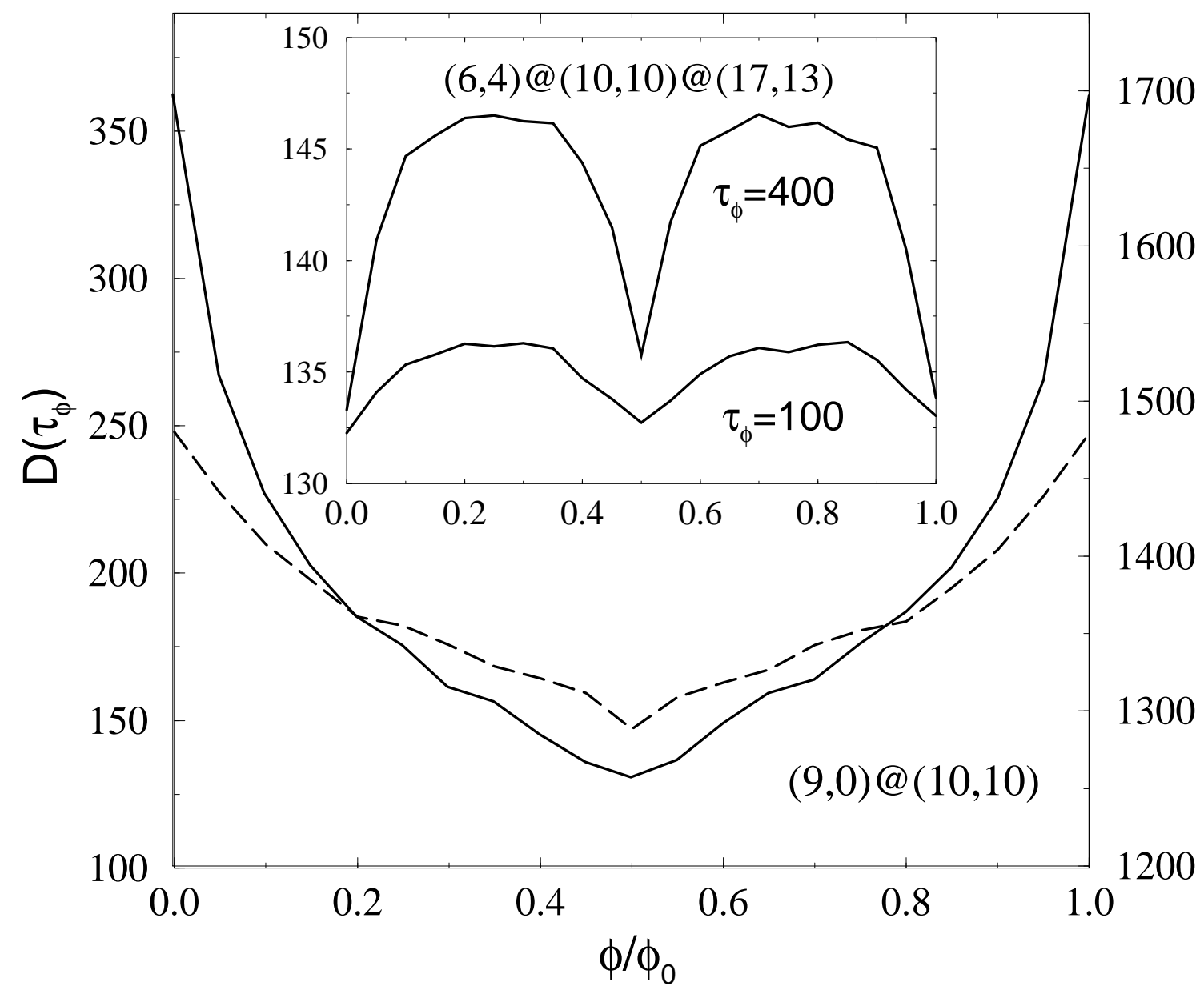




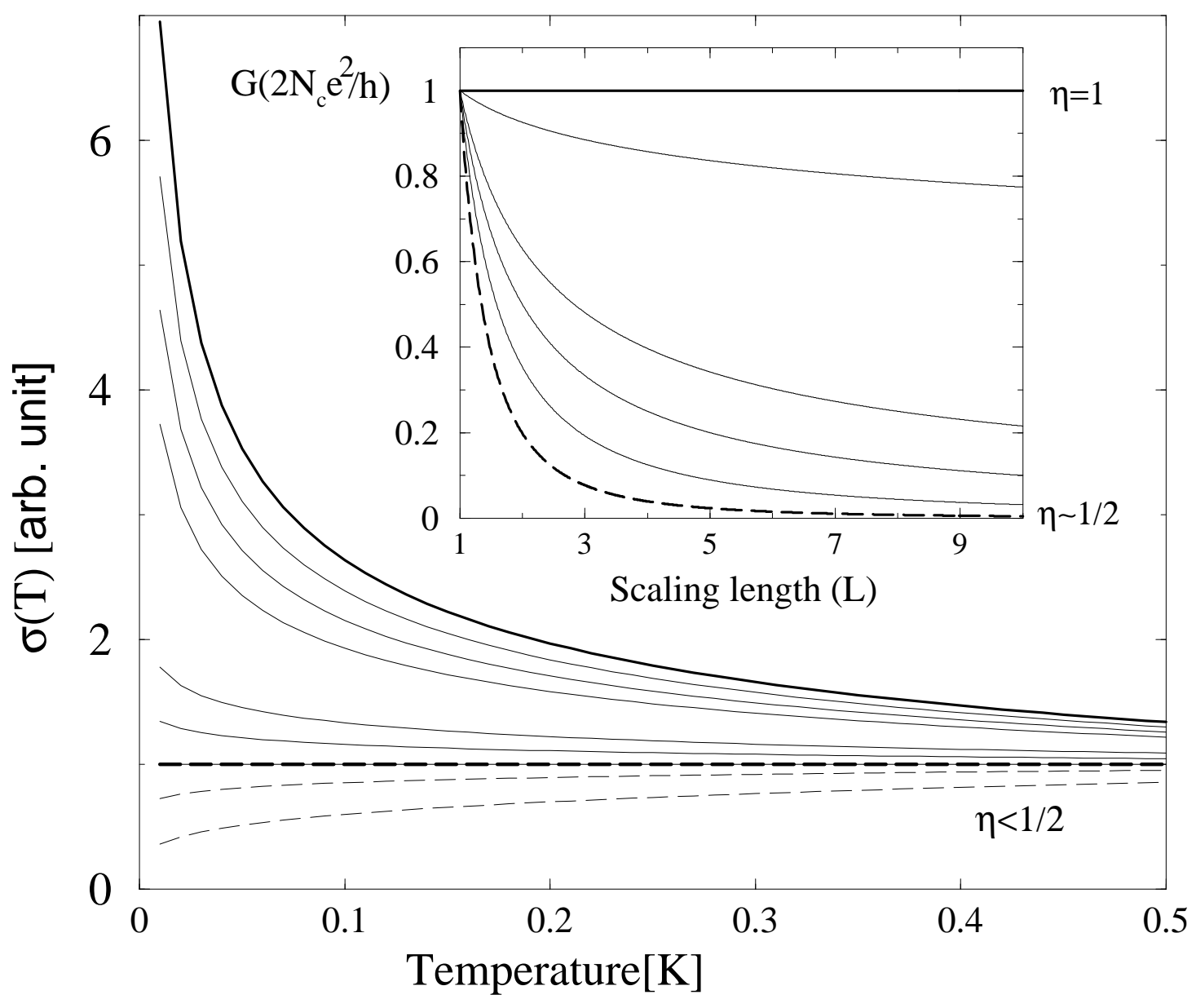

